\title{
Editorial
}

\section{Occurrence and Remediation of Pollutants in the Environment}

\author{
Núria Fontanals, ${ }^{1}$ Rathinam A. James, ${ }^{2}$ Yong Sik Ok, \\ Malini Balakrishnan, ${ }^{4}$ and Jimmy T. Efird ${ }^{5}$ \\ ${ }^{1}$ Department of Analytical Chemistry and Organic Chemistry, Universitat Rovira i Virgili, 43007 Tarragona, Spain \\ ${ }^{2}$ Department of Marine Science, Bharathidasan University, Tiruchirappalli, Tamil Nadu 620 024, India \\ ${ }^{3}$ Department of Biological Environment, Kangwon National University, Chuncheon, Gangwon-do 200-701, Republic of Korea \\ ${ }^{4}$ Energy Environment Technology Development Division, The Energy and Resources Institute (TERI), Darbari Seth Block, \\ India Habitat Centre (IHC), Lodi Road, New Delhi 110 003, India \\ ${ }^{5}$ Center for Health Disparities and College of Nursing, East Carolina University, Greenville, NC 27858, USA
}

Correspondence should be addressed to Núria Fontanals; nuria.fontanals@urv.cat

Received 14 September 2015; Accepted 15 September 2015

Copyright (C) 2015 Núria Fontanals et al. This is an open access article distributed under the Creative Commons Attribution License, which permits unrestricted use, distribution, and reproduction in any medium, provided the original work is properly cited.

In recent years, advances in human society have involved the use of large amounts and varieties of xenobiotics in various areas of our life, which certainly leads to their release into the surrounding environment. The occurrence of pollutants in air, water, and soil in turn affects biota and human health. In addition, each pollutant could undergo structural changes through various transformation and degradation pathways. In light of the possible carcinogenicity, neurotoxicity, and/or endocrine disrupting properties of some of these pollutants as well as their metabolites and transformation products, it is necessary to identify and quantify them at low concentration levels, besides identifying efficient approaches for their removal in treatment plants.

Our special issue aims at addressing novel analytical methods to determine pollutants in environment as well as develop novel strategies for their efficient removal during the treatment of polluted samples. The selected research manuscripts illustrate different research areas in the environmental field that are mainly focused on the determination, fate, and remediation of pollutants in sorted matrices.

Different kind of pollutants was treated in the studies covering from metals to pharmaceuticals or to nutrients. Thus, the sequential extraction of cadmium from soil was addressed by $\mathrm{T}$. Honma et al., who discovered the relationship between chemical forms of cadmium in soil and properties in contaminated and uncontaminated paddy soils. Veterinary antibiotics (including different tetracyclines and sulfonamides) were also determined in sediments and soil samples in the study conducted by Y. M. Awad et al. They also correlated the presence of such antibiotics with antibiotic resistance genes (ARGs), which should be further monitored to ensure public health. The release of volatile fatty acid generated, when food leachate alone or mixed with animal manure was anaerobically digested, was investigated by D.-J. Lee et al., who used the concentration of volatile fatty acid as important parameter to control and manage the anaerobic digestion.

Other studies, instead of determining different pollutants, proposed alternative strategies to remove pollutants during treatment. Thus, D. J. Lee et al. evaluated different hybrid constructed wetlands with different ventilation methods (including natural and electric ventilation) in order to enhance the nutrient removal (mainly nitrogen and phosphorous content) in conventional domestic sewage from agricultural villages. The outcomes from this study recommended an improved ventilation system via an electric fan air blower with renewable energy of solar and wind power for the nutrient removal. A. Abdel-Megeed and A. Tahir also investigated the reduction of phosphorous pollution from poultry waste by supplementing phytase enzyme in broilers fee, so that the nonrenewable inorganic phosphorous for sustainable agriculture is preserved. M. Zhang et al. tested biochar as an alternative sorbent to activated carbon (AC) for the adsorptive removal of trichloroethene (TCE). At the end, AC showed better efficiency to remove TCE from water; nevertheless, biochar is still a good alternative due to its 
cost-effectiveness. L. Zhu evaluated the performance of clean and fouled nanofiltration (NF) membranes in the rejection of organic micropollutants (particularly, polycyclic aromatic hydrocarbons, PAHs, and phthalic acid esters, PAEs). After all, suitable membrane and sample conditions were found in order to achieve an enhancement in rejection.

We believe that this special issue will be an important source of information for researchers from several disciplines covering the interdisciplinary of the environmental field.

\section{Acknowledgments}

The Guest Editors would like to thank all the authors for their valuable contributions to this special issue and the reviewers for their critical assessments of each paper.

Núria Fontanals Rathinam A. James Yong Sik Ok

Malini Balakrishnan Jimmy T. Efird 

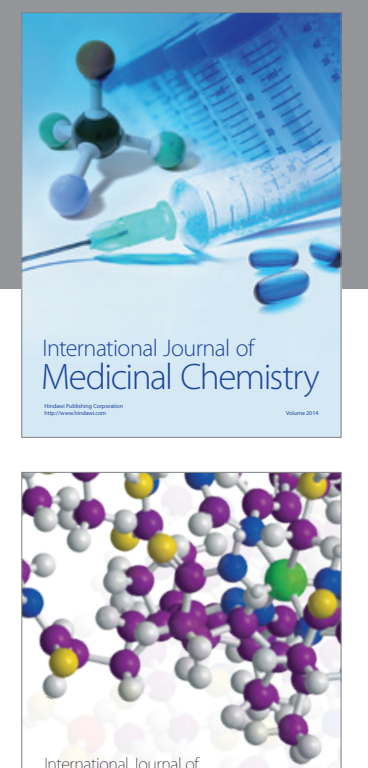

\section{Carbohydrate} Chemistry

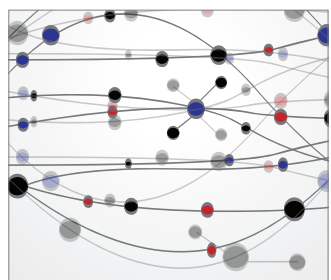

The Scientific World Journal
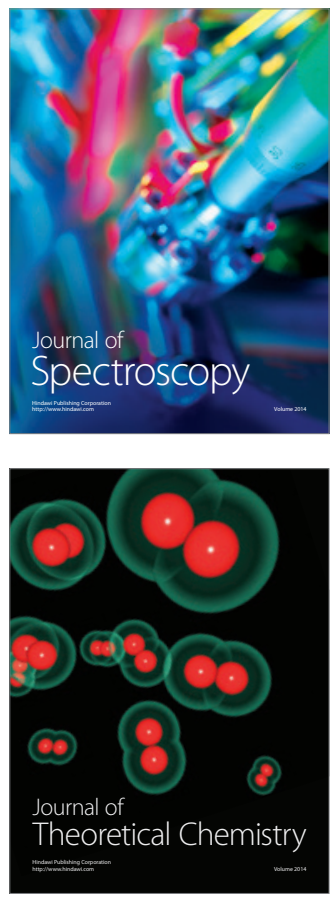
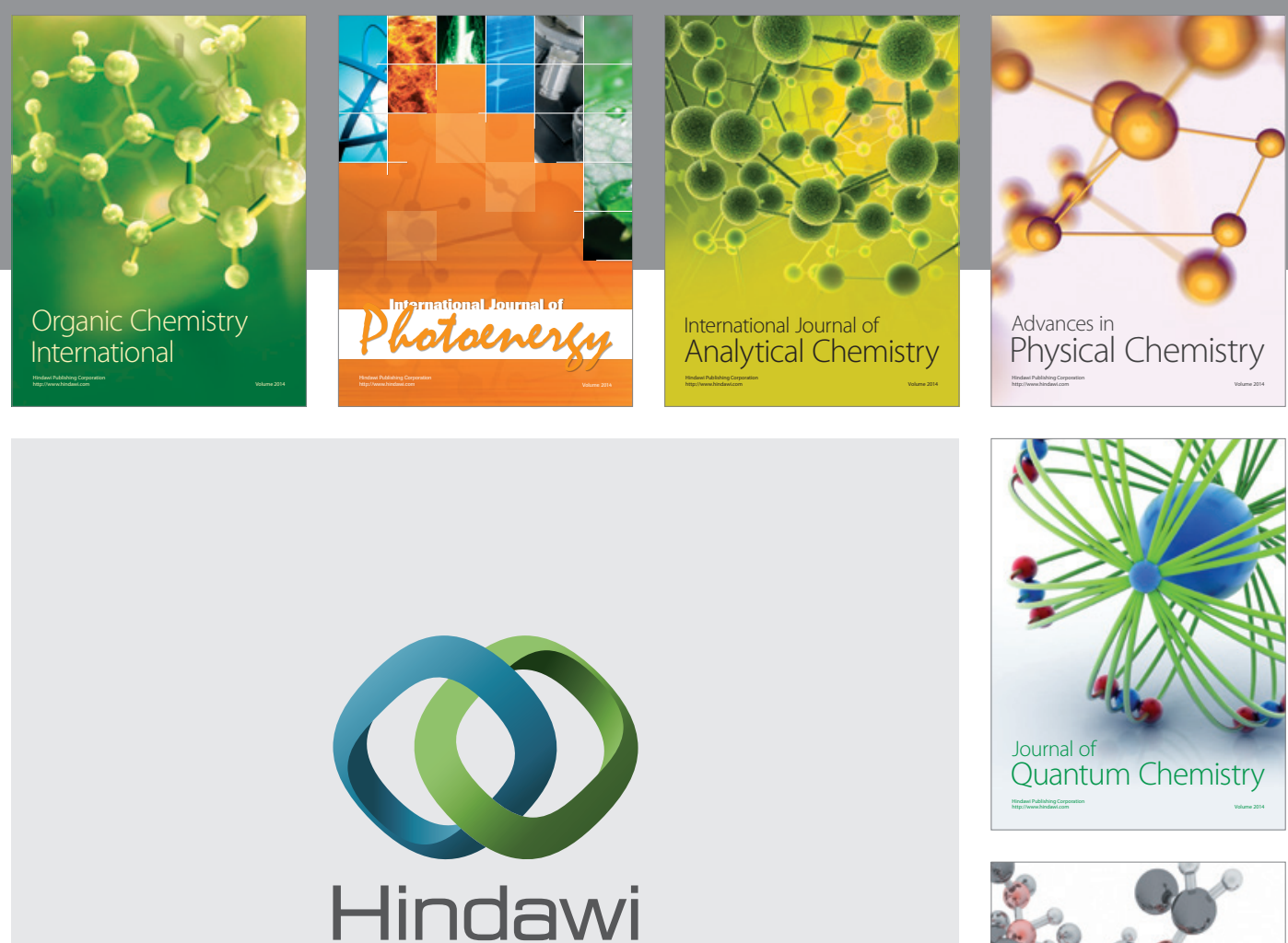

Submit your manuscripts at

http://www.hindawi.com

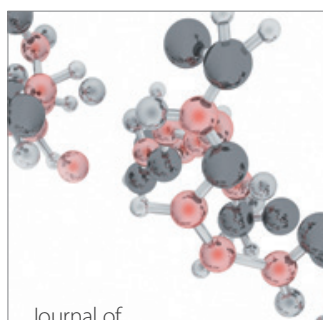

Analytical Methods

in Chemistry

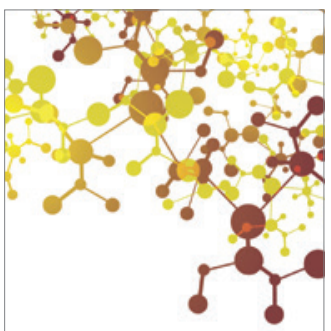

Journal of

Applied Chemistry

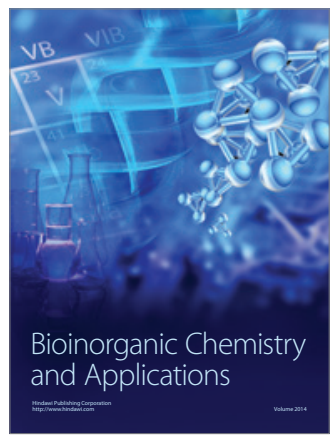

Inorganic Chemistry
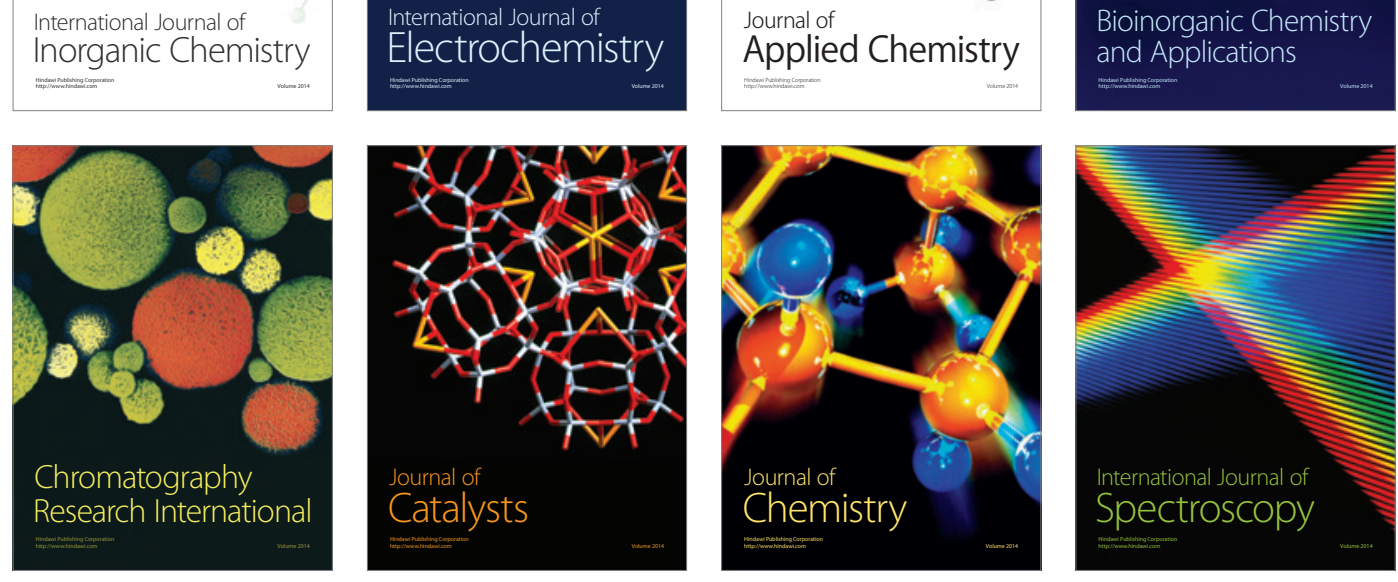